\title{
Influence of the skull conductivity perturbations on EEG dipole source analysis
}

\author{
Fangmin Chen, Hans Hallez, Peter Van Hese, Yeves D’Asseler and Ignance Lemahieu \\ Ghent University, Department of Electronics and Information Systems, Medical Image and Signal Processing (MEDISIP), \\ Ghent University Hospital-IBITECH, De Pintelaan 185 B-9000 Ghent, Belgium \\ E-mail: hans.hallez@ugent.be
}

\begin{abstract}
Electroencephalogram (EEG) source analysis is a non-invasive technique used in the pre-surgical diagnosis of epilepsy. In this study the dipole location \& orientation errors due to skull conductivity perturbations were investigated in two groups of three-dimensional (3D) head models: spherical head model and realistic head model. In each group, the head model had a brainto-skull conductivity ratio $\left(R_{\sigma}\right)$ within the range of 10 to 40 . Solving the forward problem in the head model with skull conductivity perturbations while the inverse problem in the baseline head model with $R_{\sigma}=20$ permitted to derive the dipole estimation errors. Perturbations in the skull conductivity generated dipole location \& orientation errors: the larger the perturbations, the greater the errors and the larger the error ranges. The estimated dipole locations shifted outward radially from the original location if the skull conductivity used in the forward problem was larger than that in the inverse problem $\left(\mathrm{R}_{\sigma}=10\right.$ and 15). Otherwise, the estimated dipole locations shifted radially towards the center of the head from the original location $\left(R_{\sigma}=25,30,35\right.$ and 40$)$. The dipole orientation error due to skull conductivity perturbations was not significant (maximal mean $<5$ degrees) in this study, but the dipole location error was notable (maximal mean $>5 \mathrm{~mm}$ ). Therefore, the influence of the skull conductivity perturbations on EEG dipole source analysis can not be neglected. This study suggests that it is necessary to measure the skull conductivity of the individual patients in order to achieve accurate EEG source analysis.
\end{abstract}

\section{Introduction}

Electroencephalogram (EEG) is a tool used to measure the electrical activity of groups of neurons in the brain. It records the electrical activity by measuring the potential differences between scalp electrodes as a function of time. EEG source analysis as a non-invasive tool has proven to be valuable in pre-surgical evaluation of epilepsy (Sutherling et al 1988). The focal EEG changes can be detected by EEG source analysis. This can aid neurologist to evaluate the epileptogenic zone in the brain for successful presurgical evaluation of the patients.

The electrical activity of brain tissue can be modelled by an equivalent current dipole characterised by three location parameters, two orientation angles and a magnitude. These parameters can be estimated from the measured EEG. Estimating the electrical source by this procedure is called EEG dipole source analysis. For EEG dipole source analysis both the forward and the inverse problems need to be solved. In the forward problem, the potentials at the scalp electrodes are calculated by solving the Poisson's equation for a given dipole. In the inverse problem, the potential distribution at the scalp electrodes is used to determine the dipole parameters by minimizing the relative residual energy (RRE).

To improve the technique of EEG source analysis an accurate head model is required. Skull conductivity is a crucial parameter in head model. However, to date, there is no commonly accepted value for this parameter. A wide range of skull conductivity values have been used, which are respectively 80 times (Geddes and Baker 1967, Rush and Driscoll 1968, Cohen and Cuffin 1983), 15 times (Kosterich et al 1984, Oostendorp et al 2000), 16 times (Ferree et al 2000), or 16.5 times (Hallez et al 2005) smaller than the scalp and brain conductivity. Gonçalves et al (2003) stated that the $\mathrm{R}_{\sigma}$ should be within 20 to 50 . Baysal and Haueisen (2004) suggested 23 for the in vivo brain-to-skull conductivity ratio $\left(\mathrm{R}_{\sigma}\right)$. Lai et al (2005) used cortical imaging technique to estimate in vivo human $R_{\sigma}$ and found that most $R_{\sigma}$ values are located within 18 to 34 . More recently, Zhang et al (2006) estimated the $\mathrm{R}_{\sigma}$ employing intracrancial electrical stimulation and found it was within 14 to 23 . Despite that different values of the brain-to-skull 
conductivity ratio $\left(\mathrm{R}_{\sigma}\right)$ were used in different studies, systematic study of the influence of the in vivo skull conductivity perturbations on EEG dipole source analysis has not been conducted so far.

We employed numerical approach to investigate this influence by quantitative evaluation of dipole location and orientation errors due to skull conductivity perturbations. We aimed to find a key to whether it is necessary to measure the skull conductivity of individual patients in clinic practices, in order to achieve accurate EEG dipole source analysis. We chose the skull conductivity values from the in vivo experiment results. The findings of this study will be of scientific impact on clinical practices.

\section{Methods}

\subsection{Head models}

2.1.1. Spherical head models Seven 3-shell concentric spherical head models were constructed. Each head model consisted of three compartments: a scalp, a skull and a brain compartment (see figure 1), with the radius of 92,86 , and $80 \mathrm{~mm}$, respectively.

As the white matter and the skull are anisotropic (Nicholson 1965, van den Broek et al 1998), the conductivity tensor in them could be expressed with a full matrix. The compartment was assumed to be isotropic and homogeneous throughout this study, thus, the specific tissue conductivity tensor became a scalar. The conductivity of the scalp and brain compartments was assumed to be $0.33 \mathrm{~S} / \mathrm{m}$ (Ferree et al 2000, Gonçalves et al 2003) for all the test spherical head models. The skull conductivity of the baseline spherical head model was $0.0165 \mathrm{~S} / \mathrm{m}$ (Ferree et al 2000, Gonçalves et al 2003) with $\mathrm{R}_{\sigma}=20$. The values for the $\mathrm{R}_{\sigma}$ were chosen from the in vivo experiment results (Gonçalves et al 2003, Lai et al 2005, Zhang et al 2006) and were in the range of 10 to 40. The skull conductivity values used in the seven spherical head models, were $0.033,0.022,0.0165,0.0132,0.011,0.0094$, and $0.0083(\mathrm{~S} / \mathrm{m})$ which corresponded to $\mathrm{R}_{\sigma}=10,15,20,25,30,35$ and 40, respectively.

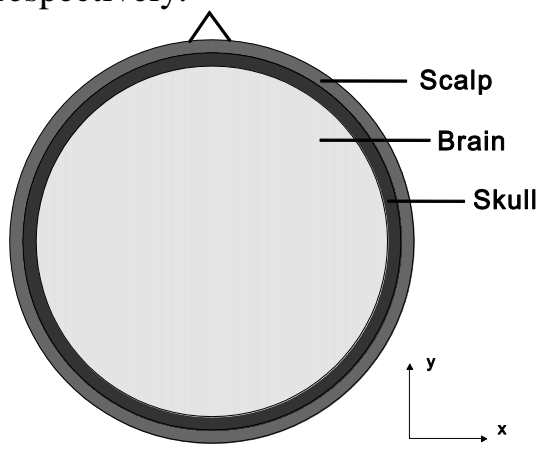

Figure 1. A 2D representation of the 3-shell concentric spherical head model, consisting of a scalp (radius 92 mm), a skull (radius 86 $\mathrm{mm}$ ) and a brain (radius $80 \mathrm{~mm}$ ) compartment.

2.1.2. Realistic head models Seven realistic head models were constructed based on the data derived from segmented T1 weighted magnetic resonant imaging (MRI). These medical images had been obtained from a 3 tesla MRI scanner (Siemens Trio, Erlangen, Germany). The realistic head model had more complex structure than the spherical head model as it incorporated more tissue types and highly irregular geometry of the head. The realistic head model consisted of a scalp, a skull, and a brain compartments. The brain compartment contained gray matter, white matter and cerebrospinal fluid (CSF). The white matter, gray matter and CSF were segmented by SPM5 from the MR images. The skull compartment, constructed by a dilation operation of the brain compartment, had an average thickness of $6 \mathrm{~mm}$. The MR images were constructed out of cubic voxels with $1 \mathrm{~mm} \times 1 \mathrm{~mm} \times 1 \mathrm{~mm}$. Extracted from these images, the realistic head model consisted of 4499621 cubic voxels, each of $1 \mathrm{~mm}^{3}$. In each cube the conductivity could be different. This cubic grid structure facilitated the solving of the forward problem (Hallez et al 2005). Three slices of a realistic head model: axial, coronal and sagittal, are depicted in figure 2 . The scalp, skull, gray matter, white matter and the CSF are represented by different colors, respectively. 


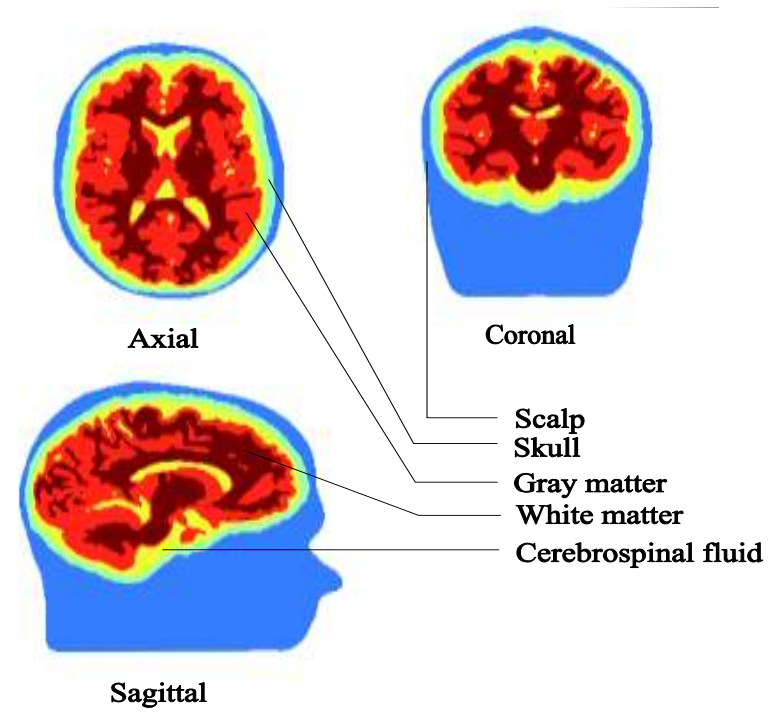

Figure 2. An axial, coronal and sagittal slice of the realistic head model. The scalp, skull, gray matter, white matter and the cerebrospinal fluid are represented by different colors, respectively.

We assumed the conductivity of all the tissues in the realistic head model to be isotropic, as in the spherical head model. The conductivity values of the skull and scalp used in the seven realistic head models were the same as those used in the spherical head models. The conductivity of gray matter and whiter matter were the same as the brain conductivity in the spherical head model. In the realistic head model the CSF conductivity was much higher than that of the gray matter and white matter, and we used $1 \mathrm{~S} / \mathrm{m}$ (Baumann et al 1997).

\subsection{Solving the forward problem}

Solving the forward problem for a given electrical source, the potentials (denoted by $\mathbf{V}$ ) at the scalp electrodes was obtained. As the electrical source was modeled by the current dipole with two infinitesimally closed monopoles (current source and sink), the current source density of the dipole could be expressed as follow:

$$
I_{\mathrm{m}}=I \delta\left(\mathbf{r}-\mathbf{r}_{2}\right)-I \delta\left(\mathbf{r}-\mathbf{r}_{1}\right),
$$

where $I_{\mathrm{m}}$ denotes the current source density of the dipole, $\mathbf{r}_{1}$ and $\mathbf{r}_{2}$ are the position coordinates of the dipole current source and sink, respectively, $\mathbf{r}$ is the location parameter of the dipole located in the middle of the two monopoles, and $\delta$ is the Dirac function. For a given current source density $I_{\mathrm{m}}$ in a volume conductor model, which defined the conductivity in the specific place, the potential $\boldsymbol{V}$ could be calculated by solving Poisson's equation as below:

$$
\nabla \cdot(\sigma(x, y, z) \cdot \nabla V(x, y, z))=I \delta\left(\mathbf{r}-\mathbf{r}_{1}\right)-I \delta\left(\mathbf{r}-\mathbf{r}_{2}\right)
$$

where $V(x, y, z)$ is the potential distribution inside the head model, $\sigma(x, y, z)$ denotes the conductivity tensor. As conductivity was assumed to be isotropic, it could be treated as a position dependent scalar.

In this study, 27 electrodes with 6 extra temporal electrodes were used, which was in conformity with the 10-20 System. This setup is used at the Department of Neurology in the Ghent University Hospital for clinical practice.

2.2.1. Solution to the forward problem in the spherical head model For the spherical head model, the forward problem was solved using the analytical formula developed by de Münck and Peters (1993), which sums up the Legendre terms. Provided that dipole position r, orientation d, and magnitude (assumed to be unity in this study) are known, the electrode potentials on the scalp can be determined by solving the forward problem which results in a $27 \times 1$ matrix.

2.2.2. Solution to the forward problem in the realistic head model For the realistic head model, no analytical solution is available. Hallez et al (2005) noted that Finite Difference Method (FDM) is suitable 
for EEG dipole source analysis in a head model with all compartments isotropic or in a head model with skull and white matter anisotropy while the grid size within $1 \mathrm{~mm}^{3}$. Moreover, Rush and Driscoll (1969) showed that the efficiency of the FDM could be improved by applying the reciprocity theorem. The grid size in our realistic head models was $1 \mathrm{~mm}^{3}$, and we used FDM with reciprocity to solve the forward problem.

\subsection{Solving the inverse problem}

By solving the inverse problem for a given scalp potential distribution, the electrical source can be estimated by finding the dipole that best fits the potential distribution. The potentials calculated at $k$ electrodes at a single time instant can be represented by $\mathbf{V}_{\text {in }} \in \mathfrak{R}^{k \times 1}$. For both the spherical and realistic head models, the dipole parameters $\left(\mathbf{r}^{\prime}, \mathbf{d}^{\prime}\right)$ were estimated by minimizing the relative residual energy (RRE) (Vanrumste et al 2000):

$$
\mathrm{RRE}=\frac{\left\|\mathbf{V}_{\text {in }}-\boldsymbol{V}\left(\mathbf{r}^{\prime}, \mathbf{d}^{\prime}\right)\right\|_{2}{ }^{2}}{\left\|\mathbf{V}_{\text {in }}\right\|^{2}{ }^{2}},
$$

here $\boldsymbol{V}\left(\mathbf{r}^{\prime}, \mathbf{d}^{\prime}\right) \in \Re^{k \times 1}$ denotes the average referenced potentials obtained from the forward calculation of equation (2) for each electrode pair and $\|.\|_{2}$ indicates the $\mathrm{L}_{2}$-norm. The solution of the dipole source localization can be found by iteratively adjusting the three location parameters with formula (3), when the RRE is minimal the optimized $\mathbf{r}^{\prime}$ is obtained. The Nelder-Mead simplex method was used to find the global minimum of the RRE, because of its simplicity (Press et al 1995). It should be noted that the relationship between the dipole components $\mathbf{d}$ and the potential $\mathbf{V}_{\text {model }}$ can be described by a matrix operator named lead-field matrix $\mathbf{L}(\mathbf{r})$. In the matrix notation we can write:

$$
\mathbf{V}_{\text {model }}=\mathbf{L}(\mathbf{r}) \cdot \mathbf{d}^{\prime} \text {. }
$$

It is easy to get the optimized $\mathbf{d}^{\prime}$ with the lead-field matrix which makes the calculation more effective.

\subsection{Simulation setup}

2.4.1. Test dipoles A 3D Cartesian coordinate system was established for each spherical head model, with the origin located at the center of the sphere. In every head model, three planes: an xy-, an xz-, and a yzplane were chosen as the test planes. In each plane, we evaluated test dipoles oriented along the $\mathrm{x}-, \mathrm{y}-$ and z- axis. Thus, for every head model 9 cases were investigated. For every case, the test dipoles were placed over the brain region at interval of $10 \mathrm{~mm}$, resulting in 193 test dipoles. In total, 1737 test dipoles for each head model and 12159 test dipoles for the seven spherical head models were investigated.

Figure 3 shows an xy-slice (top view) of the 3 -shell concentric spherical head model and the test dipoles placed in this plane. It contains 124 test dipoles in the area near the skull $(50 \mathrm{~mm}<$ radius $<80$ $\mathrm{mm})$, represented with crosses, and 69 test dipoles in the center area $(0<$ radius $<50 \mathrm{~mm})$, represented with dots.

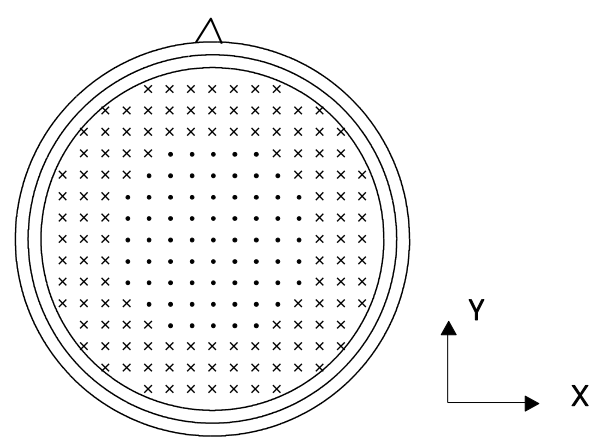

Figure 3. The test dipoles placed in an xy-plane of a 3-shell concentric spherical head model: 124 test dipoles placed in the area near the skull $(50 \mathrm{~mm}<$ radius $<80 \mathrm{~mm}$, represented with crosses) and 69 test dipoles placed in the center area $(0<$ radius $<50 \mathrm{~mm})$, represented with dots. 
In the realistic head model the neurons are in the gray matter and white matter of the brain. Hence, a great number of dipoles placed in these matters in each chosen slice were tested. For each realistic head model, three representative slices: an axial, a coronal, and a sagittal slice, which contain all the types of the tissues in the head model including large area of gray matter and white matte, were selected as the test slices. The dipole location and orientation errors in the chosen slices were investigated. In every test slice, the test dipoles along the $\mathrm{x}-, \mathrm{y}-$, and $\mathrm{z}$-axis were considered. Thus, in total 9 cases were investigated for every realistic head model. The test dipoles were placed over the gray matter and the white matter in the three test slices of every realistic head model with different $R_{\sigma}$ values.

Figure 4 illustrates the distribution of the test dipoles in the three test slices of each realistic head model. The test dipoles placed over the gray matter and white matter were presented by blue crosses and black crosses, respectively. The test dipoles were placed at interval of $10 \mathrm{~mm}$. This resulted in a total of 88536 test dipoles in the 9 cases for the seven realistic head models.

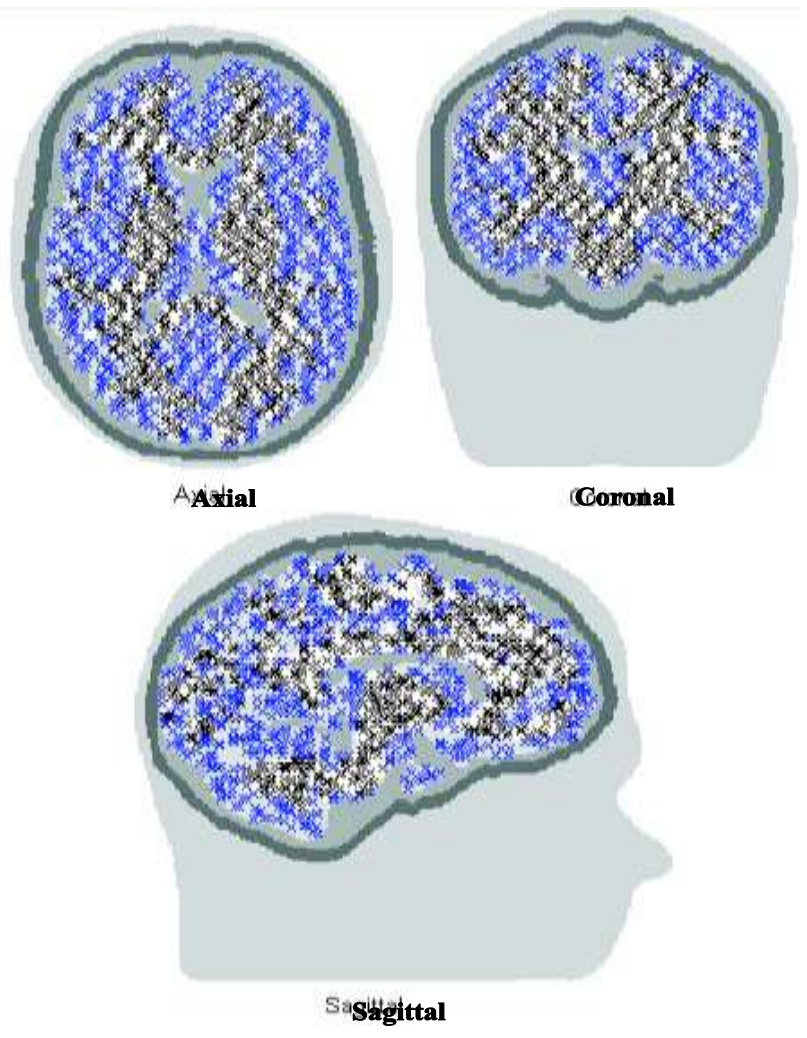

Figure 4. The test dipoles placed in each test slice of the realistic head model. The test dipoles placed over the gray matter and the white matter represented as the blue crosses and black crosses, respectively. The test slices are an axial, a coronal, and a sagittal slice in a realistic head model from T1 weight MR images.

2.4.2. Flow chart of the simulation setup Dipole estimation errors were investigated by solving the forward problem in a head model with skull conductivity perturbation while solving the inverse problem in a baseline head model with fixed skull conductivity. The simulation setup is shown in the flowchart in figure 5 .

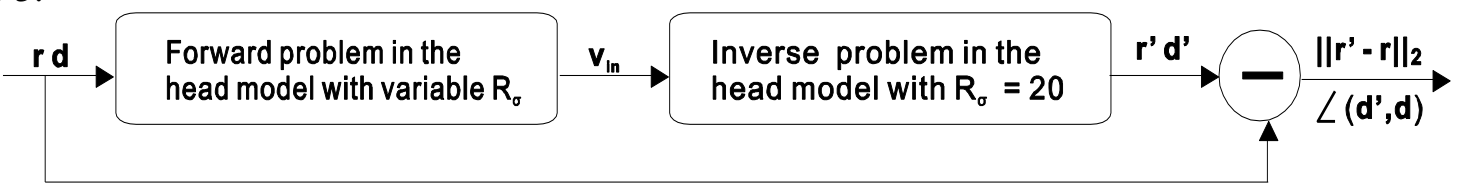

Figure 5. Flow chart of the simulation setup to investigate the dipole location and orientation errors due to a head model with skull conductivity perturbations used in the forward problem and a baseline head model with $\mathrm{R}_{\sigma}=20$ used in the inverse problem.

For each test dipole in the head model, with the dipole parameters $\mathbf{r}$ and $\mathbf{d}$ given, we solved the forward problem on the head model with variable $R_{\sigma}$ to derive the electrode potentials $\mathbf{V}_{\text {in }}$ (measured EEG). With these potentials, we were able to solve the inverse problem in the baseline head model to 
obtain the estimated dipole parameters $\mathbf{r}^{\prime}$ and $\mathbf{d}^{\prime}$. Following this procedure, the dipole estimation errors consisting of dipole location and orientation errors can be estimated.

The dipole location error was evaluated using the Euclidian Distance between the original and the estimated dipole locations, respective denoted with $\mathbf{r}$ and $\mathbf{r}^{\prime}$ :

$$
\Delta\left(\mathbf{r}^{\prime}, \mathbf{r}\right)=\left\|\mathbf{r}^{\prime}-\mathbf{r}\right\|_{2} \text {. }
$$

The dipole orientation error was evaluated by calculating the angle, using cosine rule, formed between the vector components of the original dipole $\mathbf{d}$ and the estimated dipole $\mathbf{d}^{\prime}$ :

$$
\angle\left(\mathbf{d}^{\prime}, \mathbf{d}\right)=\operatorname{acos}\left(\frac{\mathbf{d}^{\text {'T }} \mathbf{d}}{\left\|\mathbf{d}^{\prime}\right\|_{2}\|\mathbf{d}\|_{2}}\right),
$$

where $\mathbf{d}^{\mathrm{T}}$ denotes the transpose of vector $\mathbf{d}$. This simulation was repeated for each group of head models with $R_{\sigma}=10,15,20,25,30,35,40$, respectively. If the used solution schemes are accurate, then solving the forward and the inverse problems for the same head model would not generate dipole estimation error. Based on this principal, we validated the algorithms using the baseline head model with $R_{\sigma}=20$. The validated algorithm was then applied to calculating dipole estimation errors. The forward problem was solved in the head model with skull conductivity perturbations; while the inverse problem was solved in the baseline head model with constant skull conductivity which was different from that used in the forward problem. Except for the skull conductivity, all the other parameters of the head model are the same in the forward and inverse problems. Therefore, the dipole estimation errors could be completely attributed to the change in the skull conductivity. In this way, the influence of the skull conductivity perturbations on the estimated dipole errors can be determined.

\section{Results}

\subsection{Dipole estimation errors in the spherical head model}

3.1.1. Dipole location error Figure 6 presents the arrow-maps of the location error, oriented along the yaxis, of the dipoles placed in the xz-plane of the spherical head model.

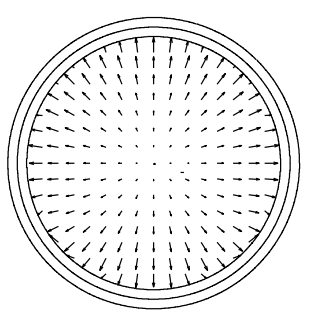

$\mathrm{R}_{\mathrm{o}}=10$

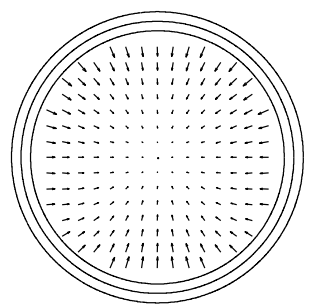

$\mathrm{R}_{\mathrm{o}}=30$

$-10 \mathrm{~mm}$

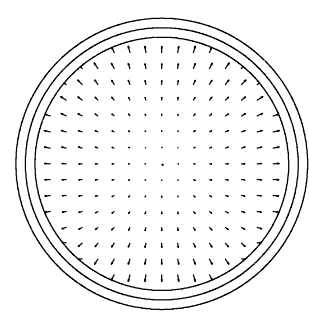

$\mathrm{R}_{\mathrm{o}}=15$

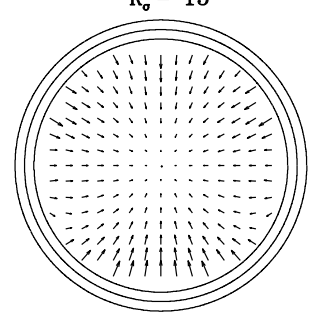

$\mathrm{R}_{\mathrm{o}}=35$

$\Delta \mathrm{Z}$

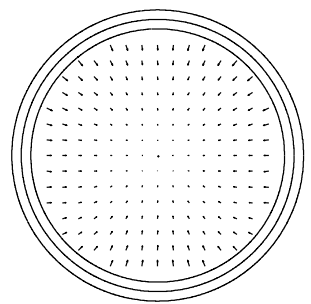

$\mathrm{R}_{\mathrm{\sigma}}=25$

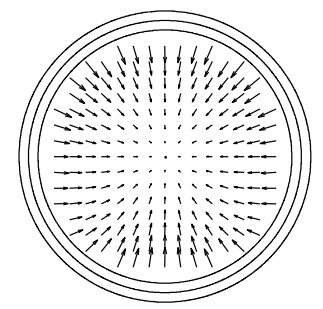

$\mathrm{R}_{\sigma}=40$

Figure 6. Dipole location error due to skull conductivity perturbations of the dipoles placed in the xz-plane oriented along the $y$-axis in each spherical head model. The arrow tail and head represent the test and the estimated dipole location projected on the xz-plane, respectively. The arrow length approximately represents the magnitude of the dipole location error on this plane. The first row displays the head models with $R_{\sigma}=10,15$ and 25 , the second row displays the head models with $R_{\sigma}=30,35$ and 40 . The horizontal line in the bottom represents a distance of $10 \mathrm{~mm}$.

The estimated dipole locations shift outward radially from the original location if the skull 
conductivity used in the forward problem is larger than that in the inverse problem (see head models with $R_{\sigma}=10$ and 15 in figure 6). Otherwise, the estimated dipole locations shift radially towards the center of the head from the original location (see head models with $R_{\sigma}=25,30,35$ and 40 in figure 6). The location error of the dipoles near the skull is greater than those in the center area. For all the head models, in each plane, the more $\mathrm{R}_{\sigma}$ deviates from the baseline value, the greater the dipole location error. The arrow-maps in the other 8 cases showing the similar features are not presented here.

Figure 7 shows the dipole location error in each spherical head model with box plot (a) and mean value (b). In this group of head models, the median and the mean of the location error and the error range increase with increased deviation of $R_{\sigma}$ from the baseline value. The mean dipole location error is larger for the dipoles placed near the skull (maximal mean $8 \mathrm{~mm}$ ) than those in the center area (maximal mean 5 $\mathrm{mm}$ ). The dipole location error over the brain is considerable with maximal mean $>5 \mathrm{~mm}$. All the maximal mean location errors occur in the head model with $R_{\sigma}=40$.

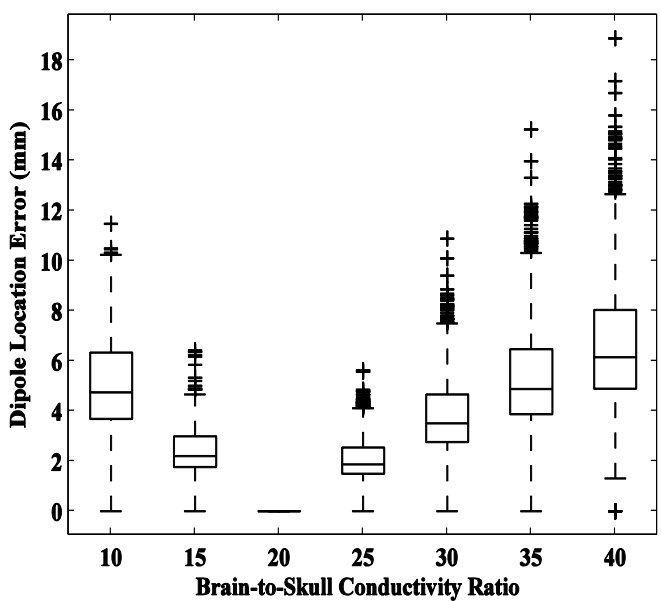

(a)

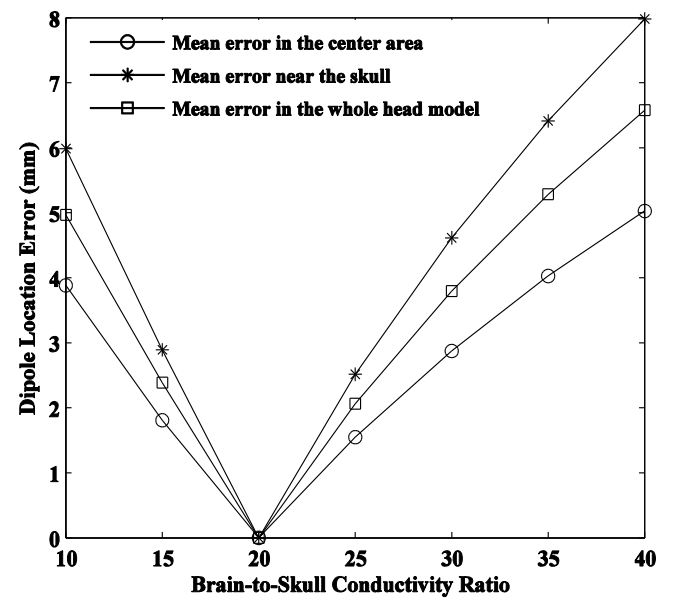

(b)

Figure 7. (a) Box plot of the dipole location error due to skull conductivity perturbations of all the dipoles investigated in the spherical head model. The horizontal axis represents the brain-to-skull conductivity ratio for the head models with $\mathrm{R}_{\sigma}=10,15,25,30,35$, and 40 and for the baseline head model $R_{\sigma}=20$. The vertical axis indicates the dipole location error in $\mathrm{mm}$. (b) The mean dipole location error due to skull conductivity perturbations in the spherical head model: the mean location error of the dipoles placed in the area near the skull $(*)$, in the center area $(0)$ and over the brain of the head model $(\square)$. The horizontal axis represents the brain-to-skull conductivity ratio for the head models with $R_{\sigma}=10,15,25,30,35$, and 40 and for the baseline head model $R_{\sigma}=20$. The vertical axis indicates the dipole location error in $\mathrm{mm}$.

3.1.2. Dipole orientation error Figure 8 shows the dipole orientation error of each spherical head model with box plot (a) and mean value (b). For all the investigated dipoles, more than $80 \%$ have an orientation error lower than 2 degrees. In the spherical head model, the median and the mean of the dipole orientation error and the error range increase with increased deviation of $R_{\sigma}$ from the baseline value. The mean orientation error is larger for the dipoles placed near the skull than those in the center area. But the orientation error is insignificant and the maximal mean is shown to be lower than 1.2 degrees.

\subsection{Dipole estimation errors in the realistic head model}

3.2.1. Dipole location error The arrow-map shows the location error of the dipoles placed in one test slice and oriented along one axis. Three representative arrow-maps, showing dipole location error in the axial slice along the $y$-axis, in the coronal slice along the $\mathrm{x}$-axis, and in the sagittal slice along the $\mathrm{z}$-axis, are presented in figure 9, figure 10, and figure 11, respectively. The dipole location error projected on the corresponding slice is greater for most dipoles placed closer to the skull. Results in the other cases not presented here also show the similar trends. 


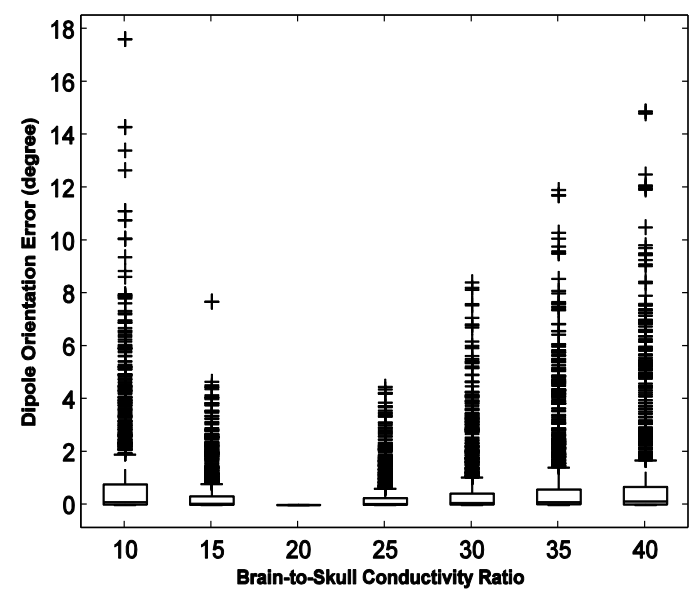

(a)

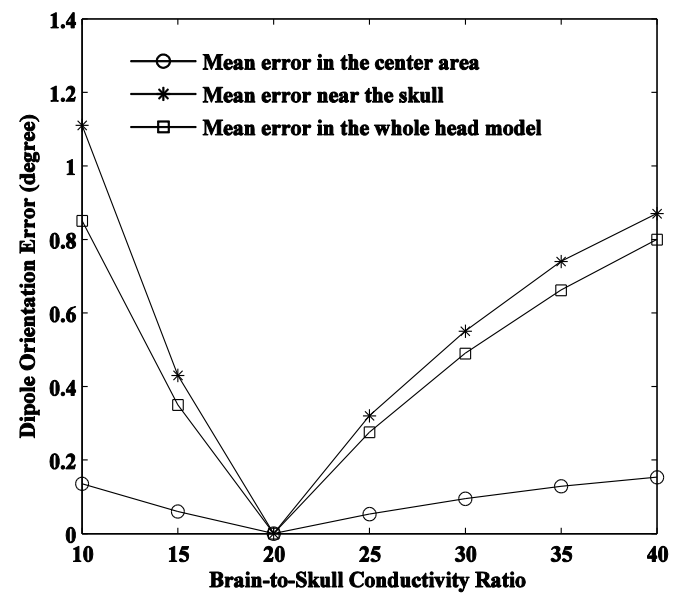

(b)

Figure 8. (a) Box plot of dipole orientation error due to skull conductivity perturbations of all investigated dipoles over the brain of the spherical head model. The horizontal axis represents the brain-to-skull conductivity ratio for the head models with $\mathrm{R}_{\sigma}=10,15,25,30,35$, and 40 and for the baseline head model $R_{\sigma}=20$. The vertical axis indicates the dipole orientation error in degrees. (b) The mean dipole orientation error due to skull conductivity perturbations in the spherical head model: dipoles placed in the area near the skull $(*)$, in the center area ( $($ ) and over the brain in each head model ( $\square$ ). The horizontal axis represents the brain-to-skull conductivity ratio for the head models with $R_{\sigma}=10,15,25,30,35$, and 40 and for the baseline head model with $R_{\sigma}=20$. The vertical axis indicates the dipole orientation error in degree.

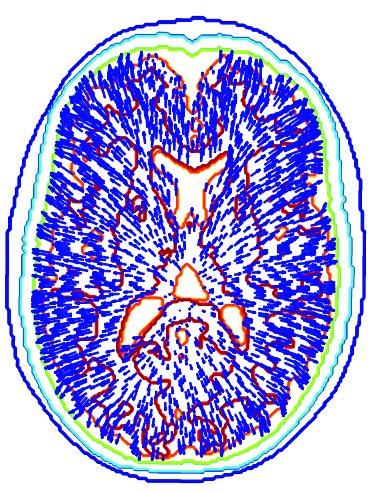

$R_{\sigma}=10$

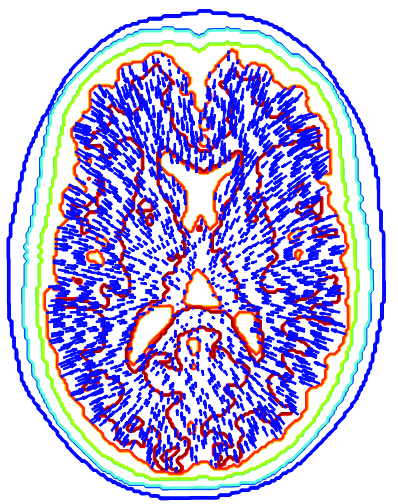

$\mathrm{R}_{\mathrm{\sigma}}=30$

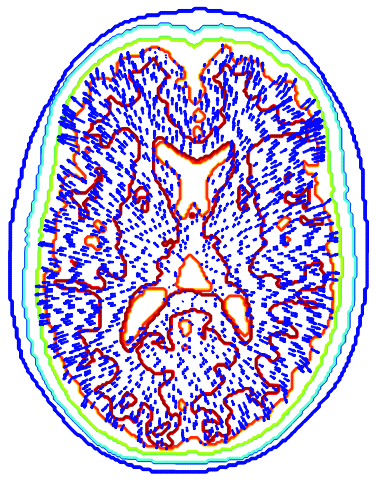

$\mathrm{R}_{\mathrm{o}}=15$

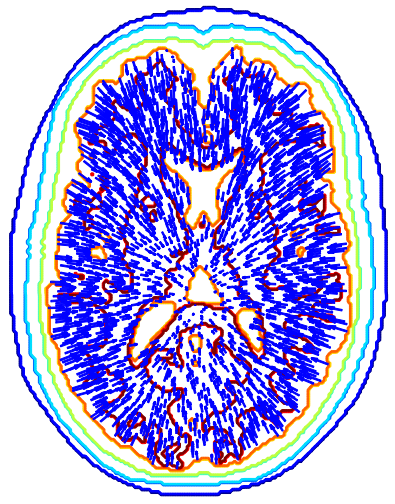

$\mathrm{R}_{\mathrm{o}}=35$

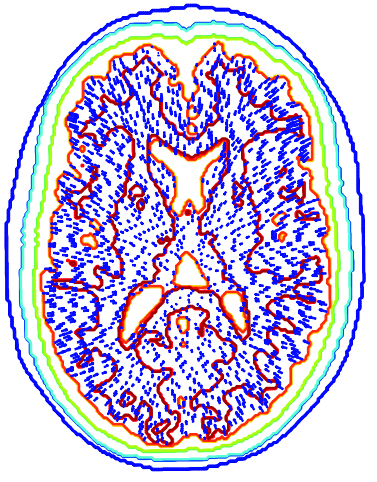

$\mathrm{R}_{\mathrm{o}}=25$

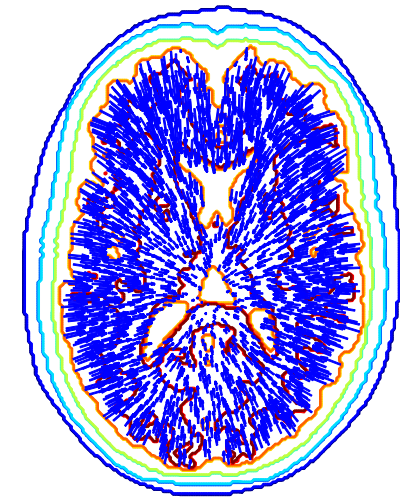

$R_{\mathrm{o}}=40$

$-\quad 10 \mathrm{~mm}$

Figure 9. Dipole location error due to skull conductivity perturbations of the dipoles placed in the axial slice oriented along the y-axis in each realistic head model. The arrow tail and head represent the test and the estimated dipole location projected on the axial slice, respectively. The arrow length represents the magnitude of the dipole location error projected on the axial slice. The first row displays the head models with $R_{\sigma}=10,15$ and 25, the second row displays the head models with $R_{\sigma}=30,35$ and 40 . The horizontal line in the bottom represents a distance of $10 \mathrm{~mm}$. 
The estimated dipole location shifts outward radially from the original location, if the skull conductivity is larger in the forward problem than that in the inverse problem (see head models with $R_{\sigma}=$ 10 and 15 in figures 9 and 10 and the first row in figure 11). Otherwise, it shifts radially towards the center of the head model from the original location (see head models with $\mathrm{R}_{\sigma}=25,30,35$ and 40 in figures 9 and 10 and the second and third rows in figure 11). For all the head models, in each slice, the more $\mathrm{R}_{\sigma}$ deviates from the baseline value, the greater the dipole location error. The similar phenomenon is observed in the arrow-maps of the other two cases for every slice.
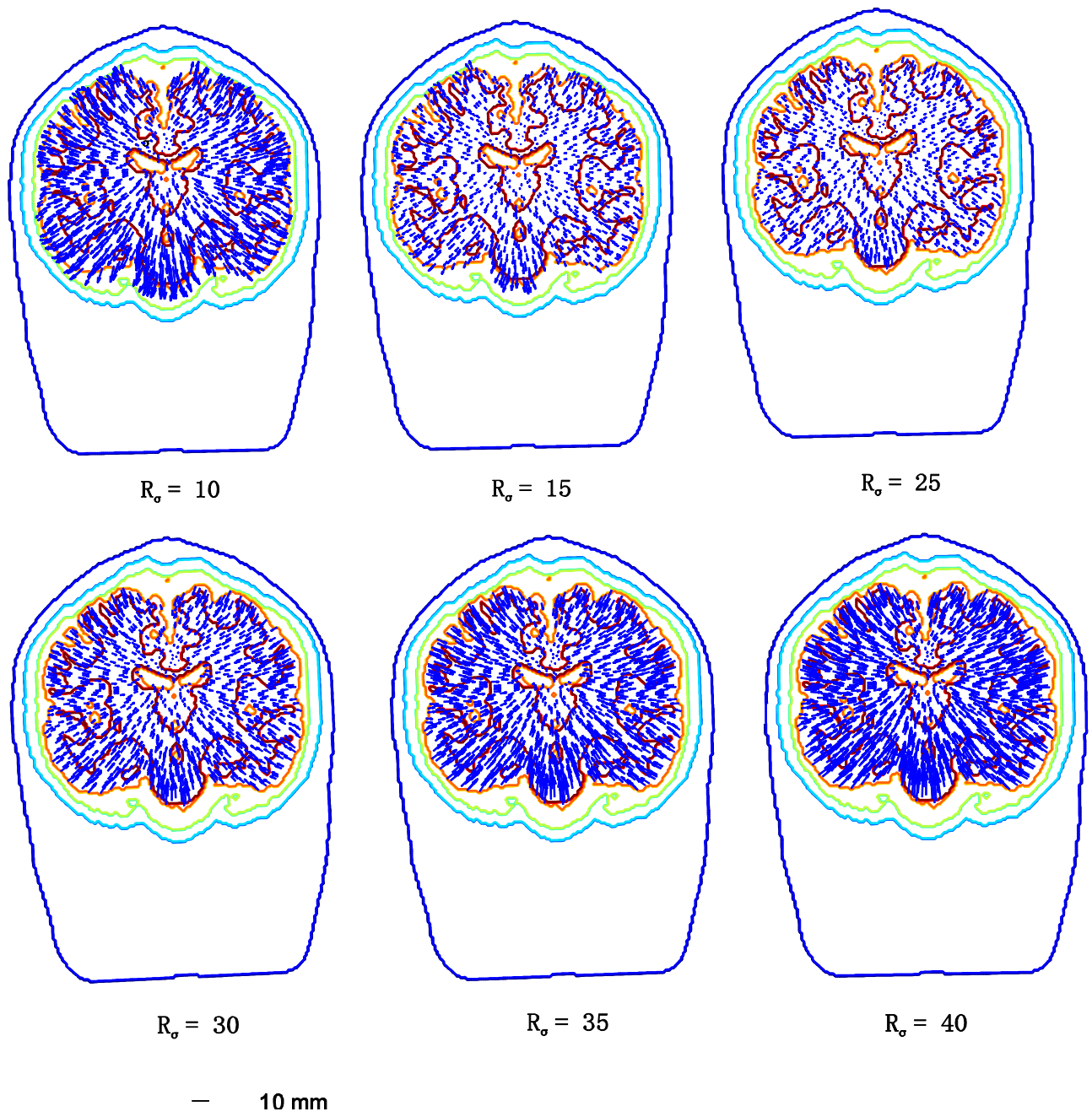

$-\quad 10 \mathrm{~mm}$

Figure 10. Dipole location error due to skull conductivity perturbations of the dipoles placed in the coronal slice oriented along the $\mathrm{x}$-axis in each realistic head model. The arrow tail and head represent the test and the estimated dipole location projected on the coronal slice, respectively. The arrow length represents the magnitude of the dipole location error projected on the coronal slice. The first row displays the head models with $R_{\sigma}=10,15$ and 25, the second row displays the head models with $R_{\sigma}=30,35$ and 40 . The horizontal line in the bottom represents a distance of $10 \mathrm{~mm}$.

Figure 12 shows the location error of all the investigated dipoles in the realistic head model with box plot (a) and mean value (b). The median and the mean of the dipole location error and the error range increase with increased deviation of $R_{\sigma}$ from the baseline value. The maximal median and error range of the dipole location error shown in the box plot occurred in the head model with $R_{\sigma}=40$. In the realistic head model, the mean dipole location error is slightly larger in the gray matter (maximal mean $6.1 \mathrm{~mm}$ ) than that in the white matter (maximal mean $5.5 \mathrm{~mm}$ ). The dipole location error over the brain is considerable with maximal mean $5.8 \mathrm{~mm}$.

3.2.1. Dipole orientation error Figure 13 shows the dipole orientation errors in the realistic head model with box plot (a) and mean value (b). The orientation error of most dipoles in the realistic head model is below 10 degrees but greater than that in the spherical head model. The median and the mean of the 
dipole orientation error and the error range increase with increased deviation of $R_{\sigma}$ from the baseline value. The maximal median and error range of the orientation error shown in the box plot occurred in the head model with $\mathrm{R}_{\sigma}=10$. The mean dipole orientation error is larger in the gray matter than that in the white matter. The dipole orientation error is higher than that in the spherical head model, with maximal mean of 4.9 degrees.
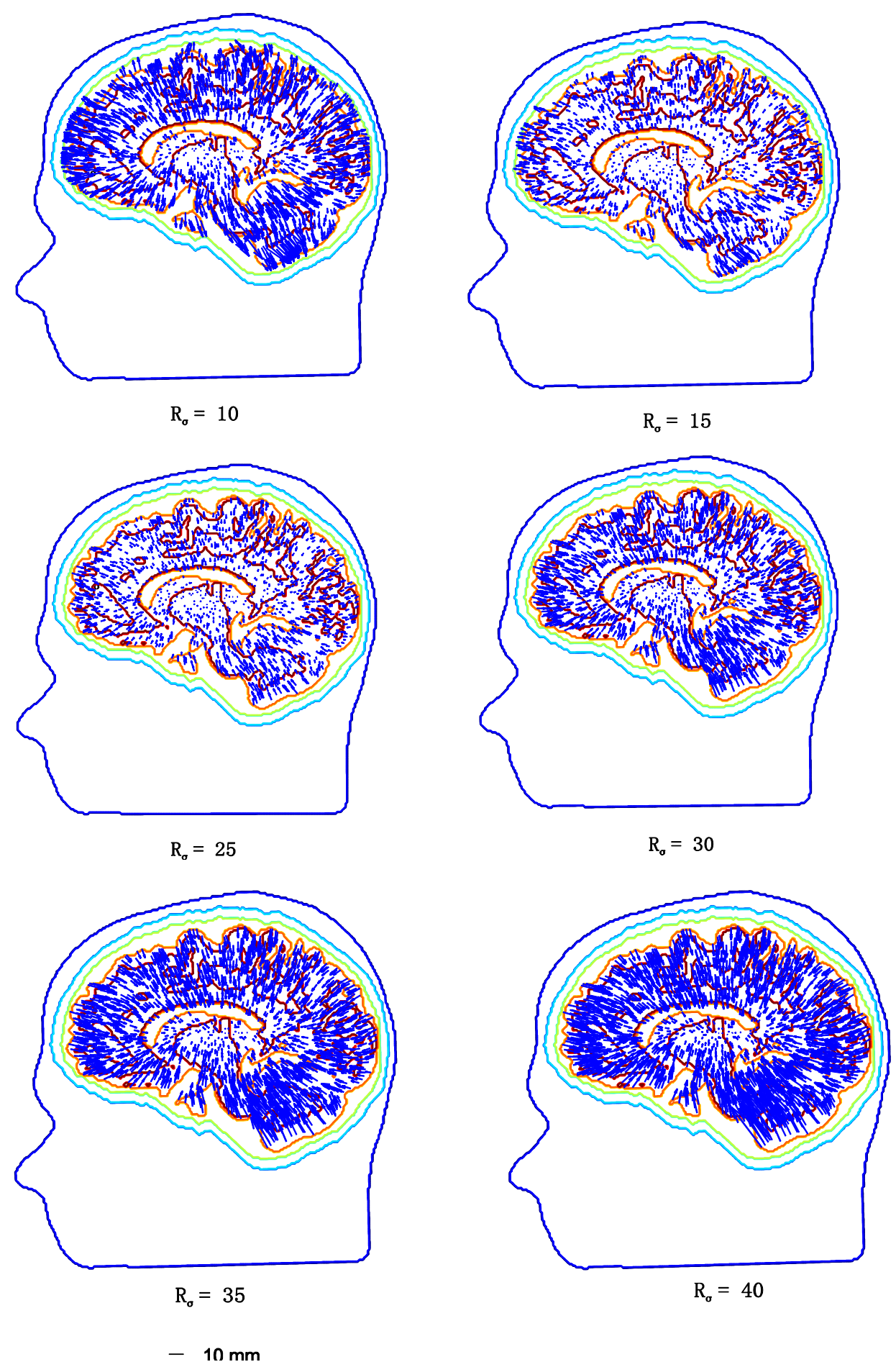

Figure 11. Dipole location error due to skull conductivity perturbations of the dipoles placed in the sagittal slice oriented along the z-axis in each realistic head model. The arrow tail and head represent the test and the estimated dipole location projected on the sagittal slice, respectively. The arrow length represents the magnitude of the dipole location error projected on the sagittal slice. The first row displays the head models with $R_{\sigma}=10,15$, the second and the third rows display the head models with $R_{\sigma}=25,30$ and $R_{\sigma}=35,40$, respectively. The horizontal line in the bottom represents a distance of $10 \mathrm{~mm}$. 


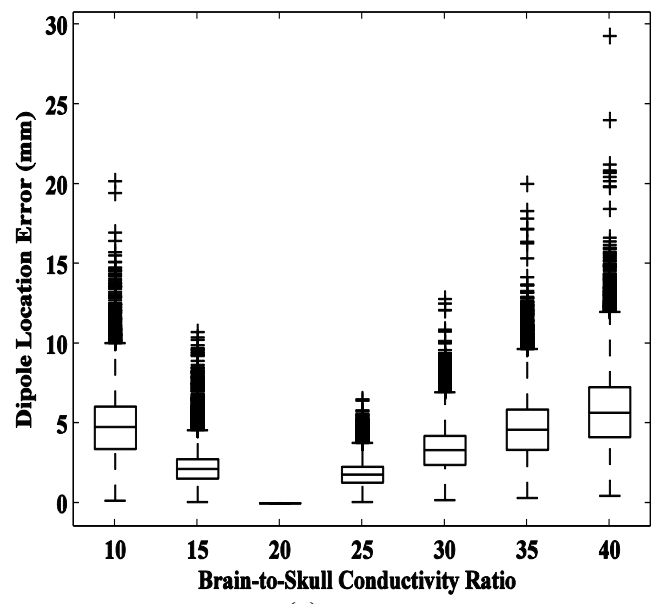

(a)

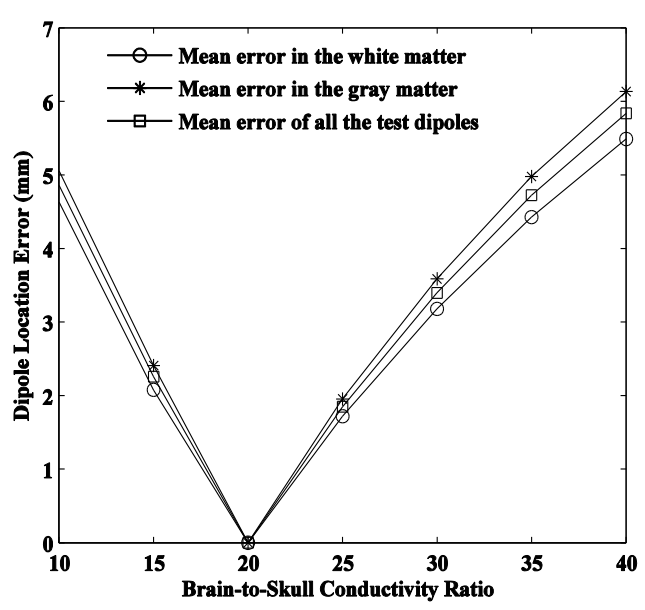

(b)

Figure 12. (a) Box plot of the dipole location error due to skull conductivity perturbations of all the investigated dipoles in the realistic head model. The horizontal axis represents the brain-to-skull conductivity ratio for the head models with $R_{\sigma}=10,15,25,30,35$, and 40 and for the baseline head model with $R_{\sigma}=20$. The vertical axis indicates the dipole location error in $\mathrm{mm}$. (b) The mean dipole location error due to skull conductivity perturbations in each realistic head model: the mean location error in the gray matter $(*)$, in the white matter $(\circ)$ and in the two matters together $(\square)$. The horizontal axis represents the brain-to-skull conductivity ratio for the head model with $R_{\sigma}=10,15,25,30,35$, and 40 and for the baseline head model $R_{\sigma}=20$. The vertical axis indicates the dipole location error in $\mathrm{mm}$.

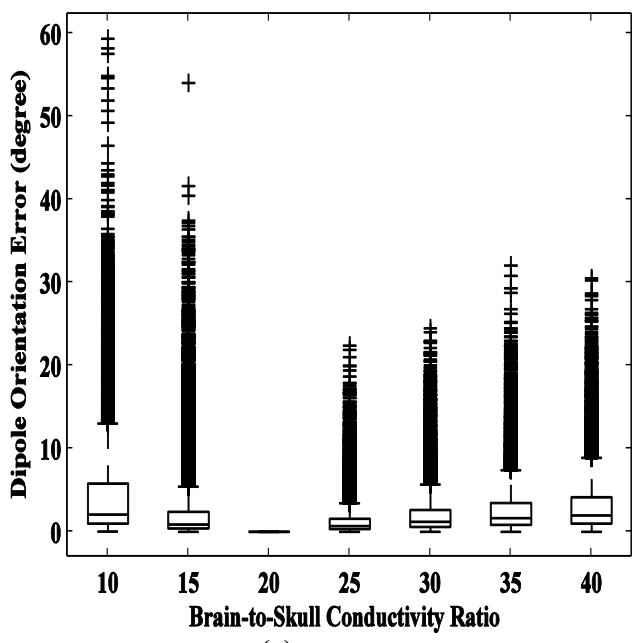

(a)

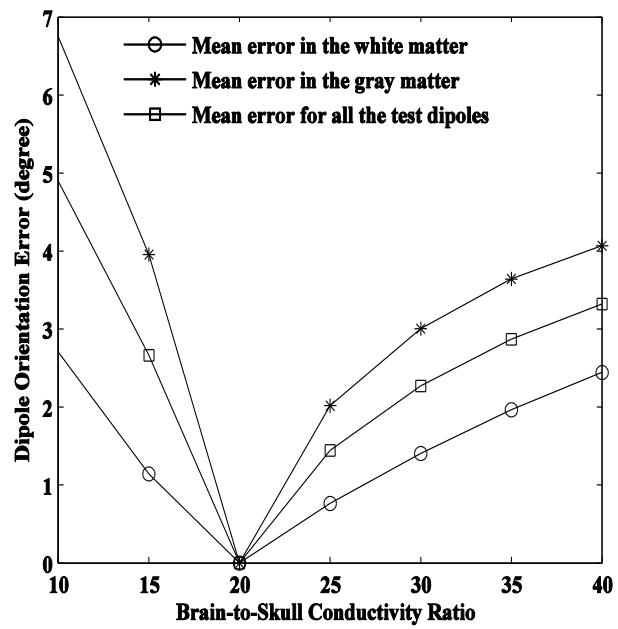

(b)

Figure 13. (a) Box plot of the dipole orientation error due to skull conductivity perturbations of all the investigated dipoles in the realistic head model. The horizontal axis represents the brain- to-skull conductivity ratio for the head models with $\mathrm{R}_{\sigma}=10,15,25,30,35$, and 40 and for the baseline head model with $R_{\sigma}=20$. The vertical axis indicates the dipole orientation error in degree. (b) The mean dipole orientation error due to skull conductivity perturbations in the realistic head models: dipoles placed in the gray matter $(*)$, in the white matter (o) and in the two matters together in the group of head models ( $\square$ ). The horizontal axis represents the brain-to-skull conductivity ratio for the head models with $R_{\sigma}=10,15,25,30,35$, and 40 and for the baseline head model with $R_{\sigma}=20$. The vertical axis indicates the dipole orientation error in degree.

\section{Discussion}

The results show that the dipole estimation errors due to skull conductivity perturbations took place in the whole volume conductor. It is observed in both the spherical and the realistic head models that, the more $\mathrm{R}_{\sigma}$ deviation from the baseline value, in other words, the larger the skull conductivity perturbation, the greater the dipole estimation errors and error range. The dipole estimation errors are generally higher in the region closer to the skull. In the realistic head model, the estimation errors of the dipoles placed in the gray matter are slightly larger than those in the white matter. This is because that more gray matter is located closer to the skull in the brain anatomy. 
The dipole location error due to skull conductivity perturbation is significant, with the maximal mean $>5 \mathrm{~mm}$ and the maximal error of $18.9 \mathrm{~mm}$ and $29.3 \mathrm{~mm}$, for the spherical and realistic head models, respectively. However, the dipole orientation error is insignificant in both groups of the head models, with the maximal average value $<5$ degrees. The estimated dipole location shifted outward radially from the original location if the skull conductivity is larger than that of the baseline head model, while it shifted inward to the center if the skull conductivity is lower.

As compared to that of spherical head models, more individual dipoles of the realistic head models have outlier dipole estimation errors in the box plot. Moreover, the realistic head models have larger maximal errors. This is because that the realistic head model has irregular geometry and contains larger number of the test dipoles (12648 test dipoles for one realistic and 1737 for one spherical head model). Except for these differences, the results from the two groups of head models agree well with each other. This study demonstrated that the findings of Chen et al. (2007), which were based on spherical head models, can be extrapolated to more complex realistic head models.

It is well known that skull conductivity perturbations have effects on the scalp potentials (Bashar et al 2009). Several researchers investigated the dipole location error due to skull conductivity perturbation (Awada et al 1998, Pohlmeier et al 1997, Laarne et al 2000, and Vanrumste et al 2000). Awada et al (1998) used 2D finite element method. In their study, the scalp potentials were measured by a discrete set of electrodes covering only the upper half of the scalp surface. They found that the dipole location error could reach $20 \mathrm{~mm}$ and the estimated dipoles shifted outward radially. Pohlmeier et al. (1997) used 65 electrodes and reported that the average dipole location error was $10 \mathrm{~mm}$ and the estimated dipole locations shifted outward radially. Later on, Laarne et al. (2000) found that the average dipole location error was about $7.5 \mathrm{~mm}$ and $6.5 \mathrm{~mm}$ when 19 electrode and 58 electrodes were used respectively. Vanrumste et al. (2000) used a large number of test dipoles and a 3D volume conductor to investigate the dipole location error due to the higher skull conductivity of head model used in the forward problem than that in the inverse problem. They found that the location error of most test dipoles was between $5 \mathrm{~mm}$ and $20 \mathrm{~mm}$, and the maximum errors were $33.4 \mathrm{~mm}$ and $28.0 \mathrm{~mm}$ for 27 and 53 electrodes, respectively. The dipole location error occurred in the entire brain volume and the estimated dipole location shifted outward radially. Besides, they noted that increasing the number of electrodes only caused slight reduction in the location error.

All the above studies evaluated only one single disturbance value: Awada et al (1998) set the skull conductivity value to be 3.3 times higher in the forward than that in the inverse problems, while Pohlmeier et al (1997), Laarne et al (2000), and Vanrumste et al (2000) set that value to 5. Furthermore, they didn't evaluate the influence of using lower skull conductivity in the forward problem than that in the inverse problem. Moreover, none of the above studies discussed the dipole orientation error. This study filled up these gaps.

This study investigated the dipole estimation errors resulting from using higher skull conductivity (head model with $\mathrm{R}_{\sigma}=10$ and 15) in the forward problem than that in the inverse problem. We found that the mean dipole location error is about $5 \mathrm{~mm}$ and $2 \mathrm{~mm}$ for the head model with $\mathrm{R}_{\sigma}=10$ and 15 used in the forward problem, respectively. These error values are lower than those shown in the discussed literatures. This is because that the deviation of the $R_{\sigma}$ from the baseline value is lower than those in the literature. Results in the two of our cases also show that the dipole estimation errors occurred in the entire brain volume and the estimated dipole location shifted outward radially from the original location.

This study also evaluated the dipole estimation errors due to using lower skull conductivity (head model with $\mathrm{R}_{\sigma}=25,30,35$ and 40) in the forward problem than that in the inverse problem. The findings suggest that the dipole estimation errors occurred in the entire brain volume and the estimated dipole location radially shifted towards the center from the original location.

This study systematically investigated the dipole estimation errors due to the skull conductivity perturbations in EEG source analysis. Clear trends of the dipole estimation errors due to skull conductivity perturbations were observed from the investigation of the two groups of head models: the larger the skull conductivity perturbation the larger the dipole estimation errors. These findings are significant as the range of the skull conductivity perturbations was set up based on in vivo estimation. The clear trends as observed in the two groups of head models provide insight into the influence of the skull conductivity perturbations and are of practical meaning to EEG application in the clinical field. Although 
the realistic head model can better present real human head than the spherical head model, it cannot characterize all the features of the human head. It would be useful to construct a more realistic head model with anisotropy in skull conductivity and study the influence of its perturbations on EEG source analysis. Huiskamp (2008) has reported that for source localization the EEG can be as accurate as MEG, if individual skull conductivity values are provided. The present study also suggests that it is necessary to measure the individual skull conductivity, in order to achieve accurate EEG source analysis.

\section{Conclusions}

We investigated the influence of skull conductivity perturbations on EEG dipole source analysis by evaluating the dipole estimation errors for both spherical and realistic head models. We conclude that:

1. The skull conductivity perturbations in the head model generate dipole location and orientation errors in the whole volume conductor. The larger the perturbation, the greater the errors and the larger the error ranges are generated.

2. The estimated dipole location shifts outward radially from the original dipole location if the skull conductivity is larger in the forward problem than that in the inverse problem, while it is radially shifted towards the center of the head model from the original dipole location if the skull conductivity is lower in the forward problem.

3. The dipoles placed closer to the skull are more sensitive to the skull conductivity perturbations and have larger estimation errors.

4. The dipole orientation error due to skull conductivity perturbations is not remarkable (maximal mean < 5 degrees), but the dipole location error is significant (maximal mean $>5 \mathrm{~mm}$ ), particularly, where the dipoles are placed near the skull.

Overall the dipole location error due to skull conductivity perturbations is considerable, so the influence of the skull conductivity perturbations on EEG source analysis is non-negligible. In the clinical application of EEG source analysis, the skull conductivity perturbation of the individual patients cannot be neglected. This study suggests that it is necessary to measure the skull conductivity of the individual patients, in order to obtain an accurate EEG source analysis.

\section{Acknowledgements}

We would like to thank Dr. J.C. de Münck for providing an implementation of the analytical formula of the spherical head model. We thank the members of MEDISIP for the continuous dedication and support. Hans Hallez is funded by a Ph.D. grant from the Institute for the Promotion of Innovation through Science and Technology in Flanders (IWT-Vlaanderen).

\section{References}

Awada K A, Jackson D R, Baumann S B, Willoams J T, Wilton D R, Fink P W and Prasky B R 1998 Effect of conductivity uncertainties and modeling errors on EEG source localization using a 2-D model IEEE transactions on biomedical engineering $4591135-45$

Bashar M R, Li Y and Wen P 2009 EEG Analysis on Skull Conductivity Perturbations Using Realistic Head Model Lecture Notes in Computer Science $5589208-15$

Baumann S B, Wozny D R, Kelly S K and Meno F M 1997 The electrical conductivity of human cerebrospinal fluid at body temperature IEEE Transactions on Biomedical Engineering 443 220-3

Baysal U and Haueisen $\mathrm{J} 2004$ Use of priori information in estimation tissue resistivities - application to human data in vivo Physiol. Meas. 25 $737-48$

Chen F, Hallez H, van Hese P, Assler Y D, Lemahieu I 2007 Dipole Estimation Errors Due to Skull Conductivity Perturbations: Simulation Study in Spherical Head Models. IEEE Proc. of Noninvasive Func. Source Imaging of the Brain and Heart and the Int. Conf. Func. Biomed. Imaging (NFSI \& ICFBI) 86-9

Cohen D and Cuffin B N 1983 Demonstration of useful difference between magnetoencephalogram and electroencephalogram Electroencephalography and Clinical Neurophysiology $5638-51$

de Münck J C and Peters M J 1993 A fast method to computer the potential in the multisphere model IEEE transactions on biomedical engineering 4011 1166-74 
Ferree T C, Eriksen K J and Tucker D M 2000 Regional head tissue conductivity estimation for improved eeg analysis IEEE Trans. Biomed. Eng. 47 1584-92

Geddes L A and Baker L E 1967 The specific resistance of biological materials - a compendium of data for the biomedical engineer and physiologist Med. Bio. Eng. 5 271-93

Gonçalves S I, de Münck J C, Verbunt J P A, Bijma F, Heethar R M and Lopes de Silva F 2003 In vivo measurement of the brain and skull resistivities using an EIT-based methods and realistic models of the head IEEE Trans. on Biomed. Eng. 50 754-67

Hallez H, Vanrumste B, Hese P V, D'Asseler Y, Lemahieu I and de Walle R V 2005 A finite difference method with reciprocity used to incorporate anisotropy in electroencephalogram dipole source localization Phy. Med. Bio. 5037873806

Huiskamp, G 2008 Interindividual variability of skull conductivity: an EEG-MEG analysis International Journal of Bioelectromagnetism $10125-$ 30

Kosterich J D, Foster K R and Pollack S R 1984 Dielectric properties of fluid-saturated bone-the effect of variations in conductivity of immersion fluid IEEE Transaction on Biomedical Engineering 31 369-73

Laarne P H, Tenhunen-Eskelinen M L and Hittinen J K, Eskola H J 2000 Effect of EEG Electrode Density on Dipole Localization Accuracy Using Two Realistically Shaped Skull Resistivity Brain Topography 124 1573-6792

Lai Y, Van Drongelen W, Ding L, Hecox K E, Towle V L, Frim D M and He B 2005 Estimation of in vivo human brain-to-skull conductivity ratio from simultaneous extra- and intra-cranial electrical potential recordings Clinical neurophysiology 116 456-65

Nicholson P W 1965 Specific impedance of cerebral white matter Experimental Neurology 13 386-401

Oostendorp T F, Delbeke J and Stegeman D F 2000 The conductivity of the human skull: results of in vivo and in vitro measurements IEEE Transactions on Biomedical Engineering 47(11) 1487-92

Pohlmeier R, Buchner H, Knoll G, Reinäcker A, Beckmann R and Pesch J 1997 The influence of skull-conductivity misspecification on inverse source localization in realistically shaped finite element head models Brain Topography 93 157-62

Press W H, Teukolsky S A, Vetterling W T and Flannery B P 1995 Numerical Recipes in C (Cambridge: Cambridge University Press) pp $430-43$

Rush S and Driscoll D A 1969 EEG Electrode Sensitivity-An Application of Reciprocity IEEE Transactions on Biomedical Engineering BME-16 $115-22$

Rush S and Driscoll D A 1968 Current distribution in the brain from surface electrodes Anesth Analgesia 47 717-23

Sutherling W W, Crandall P H, Darcey T M, Becker D P, Levesque M F and Barth D S 1988 The magnetic and electrical fields agree with intracranial localizations of somatosensory cortex Neurology 38 1705-14

van den Broek S P, Reinders F, Donderwinkel M and Peters M J 1998 Volume conduction effects in EEG and MEG Electroencephalography and Clinical Neurophysiology 1066 522-34

Vanrumste B, Van Hoey G, Van de Walle R, D’Have M, Lemahieu I and Boon P 2000 Dipole location errors in electroencephalogram source analysis due to volume conductor model errors Med. Biol. Eng. Comput. 38 528-34

Zhang Y, van Drongelen W and He B 2006 Estimation of in vivo human brain-to-skull conductivity ratio with the aid of intracranial electrical simulation Applied Physics Letters 89(22) 223903 